\title{
Respuesta de las plantas a estrés por inundación. Una revisión
}

\section{Plant responses to stress due to flooding. A review}

JUAN DE LA CRUZ JIMÉNEZ S. 1,3

LIZ PATRICIA MORENO F. ${ }^{2}$

STANISLAV MAGNITSKIY ${ }^{2}$

Corte transversal de raíz nodal de Brachiaria humidicola a 21 días de inundación.

Foto: J.A. Cardoso A.

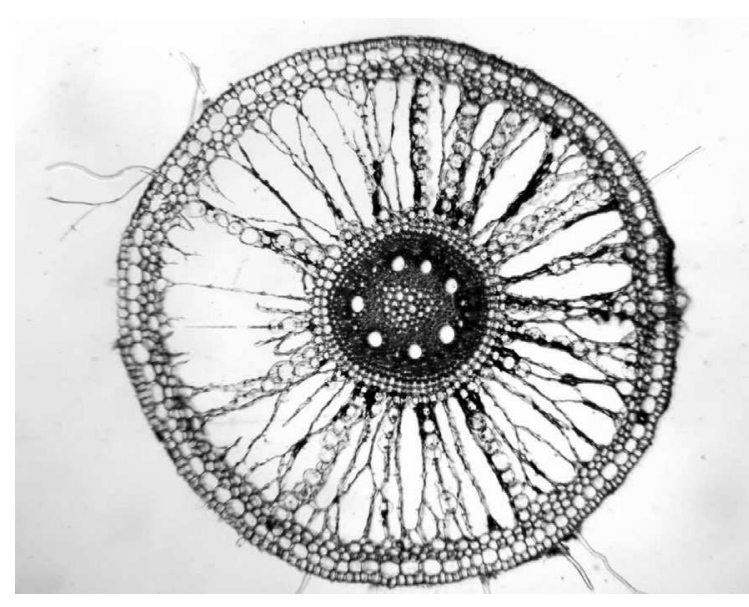

\section{RESUMEN}

La inundación tiene un efecto negativo sobre la mayoría de plantas terrestres debido a que reduce su crecimiento e induce la senescencia. La deficiencia de oxígeno, efecto principal de la inundación, cambia el metabolismo de la planta induciendo la vía anaeróbica o fermentativa como mecanismo alterno, aunque poco eficiente para la producción de energía. Igualmente el déficit de oxígeno aumenta la producción de especies reactivas de oxígeno (ROS), tanto en la mitocondria como en el cloroplasto. Como respuesta al incremento de ROS hay un aumento en el sistema de defensa antioxidante de la planta, el cual es considerado, junto con la inducción de la vía fermentativa, como una respuesta a corto plazo. Cuando la planta está sometida a largos periodos de inundación se presentan, adicionalmente, cambios morfológicos, como la formación de aerénquima, los cuales son considerados respuestas a largo plazo. En este artículo se revisan las respuestas, tanto a corto como a largo plazo, de las plantas a la condición de estrés hídrico por inundación.

Palabras clave adicionales: respiración anaeróbica, defensa antioxidante, aerénquima, estrés abiótico.

1 Facultad de Agronomía, Programa de Maestría en Ciencias Agrarias con énfasis en Fisiología de Cultivos, Universidad Nacional de Colombia, Bogotá; Centro Internacional de Agricultura Tropical (CIAT), Cali (Colombia).

2 Facultad de Agronomía, Departamento de Agronomía, Universidad Nacional de Colombia, Bogotá (Colombia).

3 Autor para correspondencia. jcjimenezser@unal.edu.co 


\section{ABSTRACT}

Flooding has a negative effect on the majority of terrestrial plants due to a reduction of growth and the resulting senescence. Oxygen deficiency, the main effect of flooding, can change plant metabolism, inducing the alternative anaerobic pathway, an inefficient mechanism for energy production. Equally, the oxygen deficit increases the production of reactive oxygen species (ROS) both in chloroplasts and mitochondria. In response to increasing ROS, plants see a rise in their antioxidant defense system, which is considered, along with the fermentation pathway, a short-term response. When plants are submitted to long periods of flooding, they present morphological changes, such as aerenchyma formation, which is considered a longterm response. In this article, we review the short and long term responses of plants to water stress caused by flooding.

Additional keywords: anaerobic respiration, antioxidant defense, aerenchyma, abiotic stress.

Fecha de recepción: 26-01-2012

Aprobado para publicación: 29-05-2012

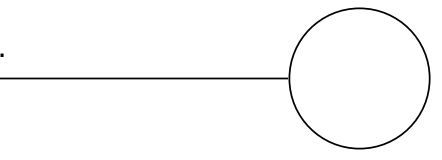

Abreviaciones: AEC: carga energética del adenilato, ACC: ácido 1-aminociclopropano-1-carboxílico, ADH: alcohol deshidrogenasa, AsA: ascorbato, APX: ascorbato peroxidasa, CAT: Catalasa, DHA: dehidroascorbato, DHAR: dehidroascorbato reductasa, GR: glutatión reductasa, GSH: glutatión, GSSG: glutatión oxidado, MDA: malondihaldehído, MDHA: monodehidroascorbato, MDHR: monodehidroascorbato reductasa, NAD: nicotinamide adenine dinucleotide, NADPH: nicotinamide adenine dinucleotide phosphate, LDH: lactato deshidrogenasa, PDC: piruvato decarboxilasa, POD: peroxidasa, ROL: (radial oxygen loss) pérdida radial de oxígeno, ROS: (reactive oxygen species) especies reactivas del oxígeno, SOD: superóxido dismutasa.

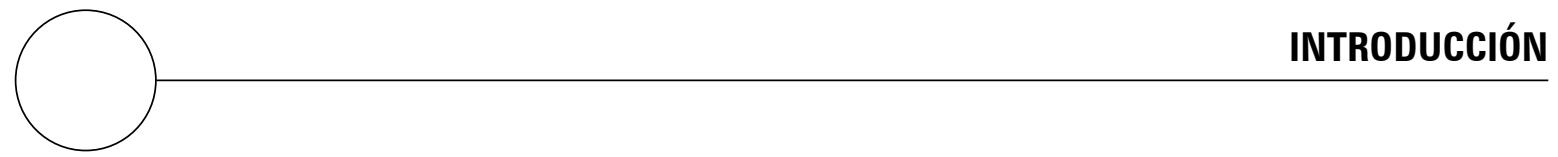

Ciertos fenómenos meteorológicos extremos, asociados al cambio climático, permiten predecir niveles elevados de vulnerabilidad de países tanto desarrollados como subdesarrollados ante sequías, ondas de calor e inundaciones (IPCC, 2007). El estrés hídrico por inundación es un factor limitante en la producción de algunos cultivos como maíz (Zea mays L.) (Zaidi et al., 2002; Bin et al., 2010; Yong-Zhong et al., 2010), brócoli (Brassica oleracea), nabo (Brassica rapa) (Issarakraisila et al., 2006), canola (Brassica napus) (Gutierrez et al., 1996; Yong et al., 2010; Leul y Zhou, 1998; Zhou y Lin, 1995), cítricos (Citrus sp.) (Hossain et al., 2009), fríjol mungo (Vigna radiata) (Sairam et al., 2008; Ahmed et al., 2006), crisantemo (Chrysanthemum sp.) (Yin et al., 2009), trigo (Triticum aestivum L.) (Dickin y Wright, 2007; Zheng et al., 2009), algodón (Gossypium hirsutum L.) (Milroy et al., 2009; Bange et al., 2003), girasol (Helianthus annuus L.) (Grassini et al., 2006), garbanzo (Cicer arietinum L.) (Palta et al., 2010), cebolla larga (Allium fistulosum L.) (Yin et al., 2009), manzana (Malus sp.) (Bai et al., 2010) y uchuva (Physalis peruviana L.) (García y Aldana, 2011).

En América Latina alrededor de 11,3\% de las tierras cultivables presentan drenajes pobres principalmente porque la fisiografía promueve inundación, niveles freáticos altos o aguas superficiales estancadas (Wood et al., 2000).

En suelos inundados, el potencial redox se disminuye (Balakhina et al., 2010 Ryser et al _2011; Abbaspour, 2012) lo cual es un indicador del bajo nivel de oxígeno presente (Unger et al., 2009a), además se afecta la disponibilidad de nutrientes para las plantas (Unger et al., 2009b). La inundación inhibe la actividad de enzimas como la $\beta$-D-glucosidasa y la fosfatasa, involucradas en 
los ciclos del carbono, nitrógeno, fósforo y azufre (Xiao-Chang y Oin, 2006) y se aumenta la concentración de etileno (Malik et al., 2003). Estas condiciones cambiantes en el suelo generan efectos negativos en el crecimiento y desarrollo de las plantas.

Cuando el oxígeno es deficiente en el suelo se generan formas reducidas de nitrógeno, fósforo, manganeso, hierro o azufre que pueden modificar la solubilidad del elemento (Unger et al., 2009b) afectando su disponibilidad (Taiz y Zeiger, 2010). La difusión de oxígeno en suelos inundados es muy baja, aproximadamente 10.000 veces más baja que en el aire (Koppitz, 2004), y el intercambio de gases entre la raíz sumergida y el ambiente es muy limitado. Bajo inundación, el aire en los poros del suelo es reemplazado por el agua y el oxígeno es consumido rápidamente por la respiración de las raíces y la actividad microbial (Koppitz, 2004).

El déficit de oxígeno inhibe la respiración mitocondrial, la oxidación y los procesos de oxigenación (Koppitz, 2004), viéndose notablemente afectado el metabolismo de la planta (Kulichikin et al., 2008; Sairam et al., 2008). El oxígeno es el aceptor de los electrones en la cadena transportadora de electrones en la mitocondria y su ausencia causa saturación de esta, por lo que se acumula $\mathrm{NADH}$ disminuyéndose la producción de ATP (Lin et al., 2008). En ausencia del aceptor de electrones, la oxidación de $\mathrm{NADH}$ se bloquea y no se genera el $\mathrm{NAD}^{+}$necesario para glicolisis, esto disminuye la producción de la energía necesaria para soportar los procesos metabólicos de la planta (Taiz y Zeiger, 2010).

Como respuesta al estrés por inundación en la planta se inducen vías de señales de transducción que llevan al desarrollo de la vía metabólica de la fermentación y posteriormente, de acuerdo con la especie, a la generación de cambios morfológicos y formación de raíces adventicias (Yin et al., 2010).
Los cambios bioquímicos en las plantas son usualmente inducidos por un periodo corto de inundación mientras que los cambios anatómicos y morfológicos están involucrados con aclimatación a largos periodos de inundación (Chen et al., 2002). En esta revisión se presentan las adaptaciones de las plantas tanto a corto como a largo plazo en respuesta al estrés por inundación.

\section{RESPUESTA BIOQUÍMICA}

Las plantas expuestas a condiciones anaeróbicas pueden cambiar su metabolismo de respiración aeróbica a la vía de la fermentación, como un mecanismo adaptativo a la carencia de oxígeno (Fukao et al., 2003; Peña-Fronteras et al., 2008). En ausencia de oxígeno, el ciclo del ácido cítrico y la fosforilación oxidativa no funcionan y la glicolisis se detiene por la limitación en el contenido de $\mathrm{NAD}^{+}$. Cuando se agota el NAD ${ }^{+}$, necesario para la primera reacción de la fase de conservación de energía de la glicolisis, la planta metaboliza piruvato por la vía fermentativa para obtener $\mathrm{NAD}^{+}$(Taiz y Zeiger, 2010). Las plantas realizan dos tipos de fermentación: alcohólica y del ácido láctico, estas dos vías metabólicas utilizan como substrato el piruvato, producido en la glicolisis (Kulichikhin et al., 2008) (figura 1). En la fermentación del ácido láctico, la enzima lactato deshidrogenasa (LDH) utiliza $\mathrm{NADH}$ para reducir piruvato a lactato y regenerar $\mathrm{NAD}^{+}$(Kato-Noguchi, 2000; Taiz y Zeiger, 2010). Lo mismo ocurre en la fermentación alcohólica, donde la enzima alcohol deshidrogenasa $(\mathrm{ADH})$ utiliza NADH para reducir acetaldehído a etanol y regenerar $\mathrm{NAD}^{+}$. La acumulación de lactato causa una disminución del pH citoplasmático (Kato-Noguchi, 2000; Taiz y Zeiger, 2010), lo que es común en plantas susceptibles a la anoxia (Couldwell et al., 2009). Cuando se acidifica el citoplasma, se suprime la síntesis de proteínas, se detiene la glicolisis y se presenta un déficit extremo de energía en la planta (Kulichikhin et al., 2008). 
La disminución en el $\mathrm{pH}$ inhibe la actividad de la LDH (Kulichikhin et al., 2008) y activa la ADH y la piruvato decarbixilasa (PDC). La enzima PDC cataliza la conversión de piruvato a acetaldehído en el primer paso en la vía de la fermentación alcohólica (Yin et al., 2009). Luego el acetaldehído es reducido a etanol en una reacción catalizada por la $\mathrm{ADH}$, donde el $\mathrm{NADH}$ es oxidado (figura 1) (Kato-Noguchi et al., 2000; Peña-Fronteras et al., 2008), la producción de etanol es menos tóxica para la planta ya que este puede difundir fuera de la célula (Taiz y Zeiger, 2010). La fermentación etanólica no necesita ser precedida por la fermentación del lactato (Kato-Noguchi et al., 2000; Mohanty y Ong, 2001) y el factor más limitante para esta vía, bajo condiciones de déficit de oxígeno, no es la $\mathrm{ADH}$ sino la cantidad reducida de PDC (Kato-Noguchi et al., 2000).

La cantidad de ATP generada durante la fermentación es de 2 moléculas de ATP por molécula de glucosa, producidas por fosforilación de substrato (Stryer, 1995; Aschi-Smiti, 2003; Taiz y Zeiger, 2010), mientras que durante la fosforilación oxidativa se producen 32 moléculas de ATP (Mohanty y Ong, 2001). La baja producción de energía en la fermentación se debe a que mucha de la energía disponible en la sacarosa es conservada en el producto, lactato o etanol (Taiz y Zeiger, 2010). Dada la baja eficiencia de generación de energía por la fermentación, es necesario un incremento en la tasa de la glicolisis para producir el ATP que necesita la célula para sobrevivir (Taiz y Zeiger, 2010).

El aumento en la tasa glicolítica contribuye a mejorar el estado energético de la planta y el uso eficiente de los carbohidratos para la producción de energía (Aschi-Smiti, 2003). Dado que la fermentación utiliza la glucosa como sustrato, un adecuado suministro de azúcares fermentables, un aumento en la degradación de almidón o el almacenamiento de carbohidratos como azúcares simples, puede ser un mecanismo importante para sobrevivir a las condiciones de anegamiento (Peña-Fronteras et al., 2008). Igualmente, la disponibilidad de carbohidratos fermentables aparece como un parámetro bioquímico relacionado con la tolerancia a inundación en cereales (Guglielminetti et al., 2001) ya que estos son utilizados por la planta bajo anoxia. Durante el primer día de inundación, el nivel de los azúcares fermentables disminuye (Guglielminetti et al., 2001), pero posteriormente este aumenta probablemente por la degradación de almidón (Guglielminetti et al., 2001).

Las plantas de trigo, cebada y arroz presentan una alta producción de etanol durante el primer día de inundación, de estas solo el arroz tiene la capacidad para fermentar durante periodos largos de anoxia (Guglielminetti et al., 2001). El primer día de anoxia, la tasa de producción de etanol en plántulas de arroz es cercana a 2 (conversión completa de carbohidrato a etanol, 2 moles de etanol por mol de hexosa), después incrementa rápidamente a valores que no son comparables con los cambios en el contenido de carbohidratos, en contraste en trigo y cebada es menos de 2 moles de etanol por mol de hexosa (Guglielminetti et al., 2001).

Los cambios en la ruta metabólica se producen rápidamente después del estrés por inundación. En plantas de lechuga, se encontró un aumento en la concentración de etanol y acetaldehído después de 30 min de anoxia, el etanol a las primeras 6 h incrementa ocho veces más que en condiciones aeróbicas, mientras que la actividad de la $\mathrm{ADH}$ y la PDC aumenta en 2,8 y 2,9 veces respectivamente, más que en condiciones de normal aireación o normoxia (Kato-Noguchi et al., 2000). En genotipos de fríjol mungo tolerantes a la inundación, se observan incrementos de 15 a 20 veces en la actividad de la $\mathrm{ADH} 8 \mathrm{~d}$ después de la inundación, sin embargo los genotipos susceptibles solo presentan un aumento a los 4 d de inundación y después una disminución de la actividad (Sairam et al., 2008). En genotipos de Dendranthema nankingense y D. zawadskii tolerantes a inundación la actividad de la $\mathrm{ADH}$ fue el doble en comparación con los genotipos 
susceptibles, en contraste la actividad de la LDH fue tres veces mayor en las especies susceptibles durante un periodo de $6 \mathrm{~d}$ de inundación (Yin et al., 2010). La actividad de la ADH y la PDC en rizomas de Cyperus rotundus aumenta significativamente cuando se someten a condiciones de hipoxia por 24 h después de la germinación (Peña-Fronteras et al., 2008).

\section{RESPUESTAS DEL SISTEMA ANTIOXIDANTE DE LA PLANTA}

El estrés por inundación genera en la planta cambios que pueden ser irreversibles como disminución de la permeabilidad de las membranas, peroxidación de lípidos, degradación de proteínas clorofilas, disminución de la expansión foliar y cierre estomático. El cierre estomático causa dis- minución del $\mathrm{CO}_{2}$ en los espacios intercelulares y disminución en la fotosíntesis (Lin et al., 2006), esto induce la formación de las especies reactivas de oxígeno (ROS) (Mittler, 2002).

La sobreproducción de ROS bajo estrés por inundación está directamente relacionada con la disminución en la tasa fotosintética bajo esta condición de estrés (Hossain et al., 2009), ya que menor asimilación de $\mathrm{CO}_{2}$ causa un desacople entre las reacciones del tilacoide y el ciclo de Calvin (Lin et al., 2008). Las ROS son formas parcialmente reducidas del oxígeno atmosférico $\left(\mathrm{O}_{2}\right)$ y se pueden generar por la excitación del $\mathrm{O}_{2}$ a la forma simple $\left({ }^{1} \mathrm{O}_{2}\right)$ o por la transferencia de uno, dos o tres electrones de la forma $\mathrm{O}_{2}$ al radical superóxido $\left(\mathrm{O}_{2}^{-}\right)$, peróxido de hidrógeno $\left(\mathrm{H}_{2} \mathrm{O}_{2}\right)$ o al radical hidroxilo $\left(\mathrm{OH}^{-}\right)$respectivamente (Lin et al., 2008; Mittler, 2002).

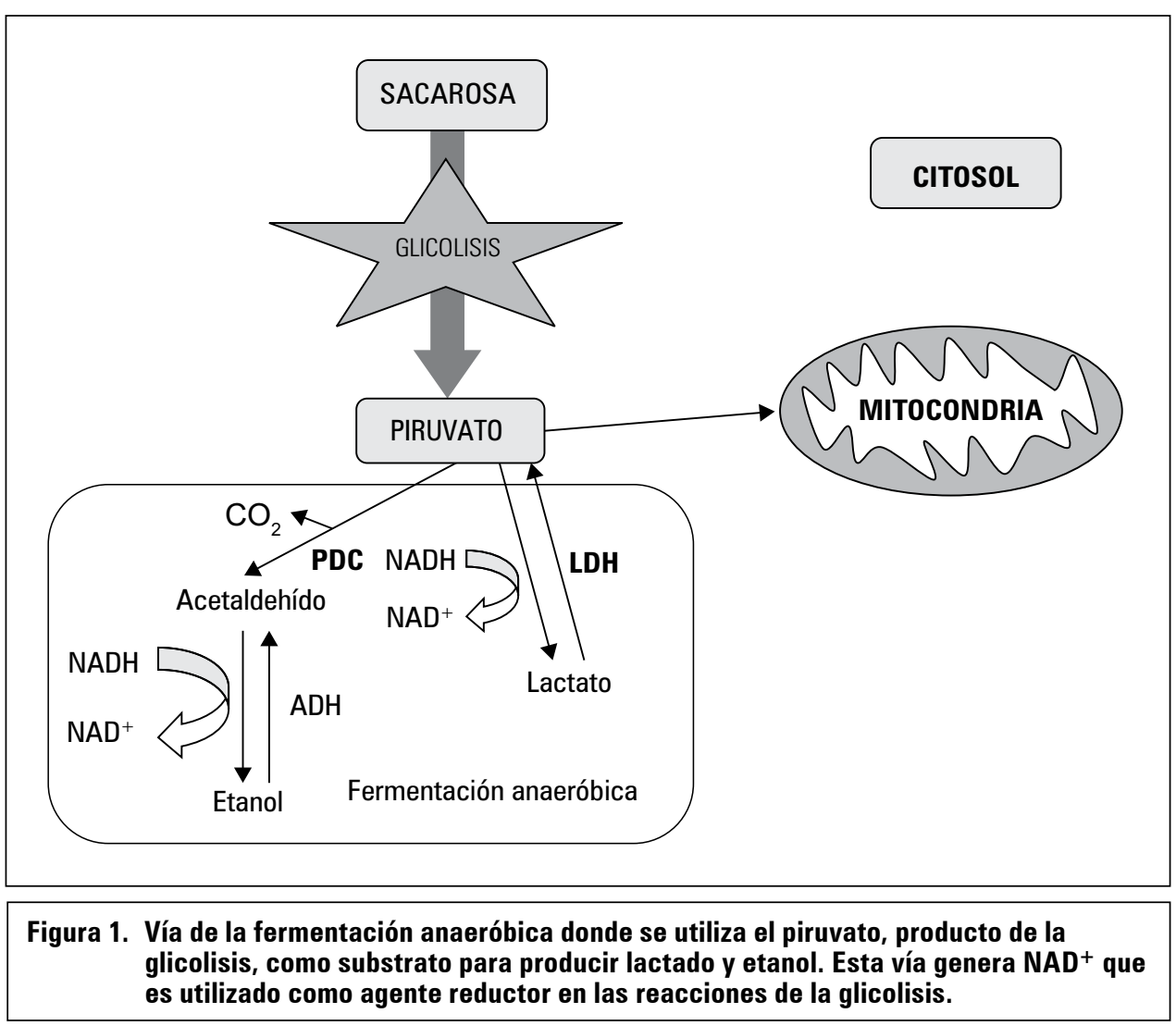

REV. COLOMB. CIENC. HORTIC. 
Las ROS son un indicador celular de estrés, un mensajero involucrado en respuestas a estrés (Mittler, 2002; Fukao y Bailey-Serres, 2004; Cheeseman, 2007; Lin et al., 2008) y además está involucrado en la muerte celular bajo estrés abiótico (Evans, 2003). Las ROS se generan en compartimentos celulares y su producción es resultado del metabolismo en todos los organismos vivos (Mubarakshina et al., 2006). En plantas son producidas en cloroplastos, mitocondrias y peroxisomas, durante estrés la tasa de producción se incrementa notablemente (Suzuki y Mittler, 2006). Las ROS pueden causar mutaciones, disfunción de proteínas, oxidación de lípidos, daños en membranas, inhibición de la síntesis de clorofila y, por tanto, disminución en la tasa fotosintética (Ella et al., 2003).

Las plantas presentan compuestos antioxidantes enzimáticos y no enzimáticos que les permiten contrarrestar los efectos fitotóxicos causados por ROS (Alscher et al., 1997). Los antioxidantes enzimáticos son: la superóxido dismutasa (SOD), la ascorbato peroxidasa (APX), la catalasa (CAT) (Yin et al., 2010; Yordanova et al., 2004), la monodehidroascorbato reductasa (MDHAR), la dehidroascorbato reductasa (DHAR), la glutatión reductasa (GR) (Hossain et al., 2009; Ella et al., 2003) y la glutatión peroxidasa (GPX). Los antioxidantes no enzimáticos incluyen los carotenoides, el ascorbato (AsA) (Bin et al., 2010), el glutatión (GSH) (Bai et al., 2010), los compuestos fenólicos y los tocoferoles (Yordanova et al., 2004).

La SOD representa el primer nivel de defensa contra los radicales $\mathrm{O}_{2}^{-}$(Hossain et al., 2009) a través de la formación de $\mathrm{H}_{2} \mathrm{O}_{2}$ y $\mathrm{O}_{2}$ (Yordanova et al., 2004; Bin et al., 2010), el $\mathrm{H}_{2} \mathrm{O}_{2}$ es menos tóxico y es convertido en $\mathrm{H}_{2} \mathrm{O}$ por una serie de reacciones subsecuentes (Bin et al., 2010). La APX es la primer enzima que elimina $\mathrm{H}_{2} \mathrm{O}_{2}$ (Bai et al., 2010) para producir $\mathrm{H}_{2} \mathrm{O}$, la $\mathrm{POD}$ puede estar involucrada en la eliminación de $\mathrm{H}_{2} \mathrm{O}_{2}$ y $\mathrm{OH}^{-}$(Yong-Zhong et al., 2010). Igualmente, la enzima CAT está involucrada en la regulación de los niveles intracelulares de $\mathrm{H}_{2} \mathrm{O}_{2}$ (Bin et al., 2010) convirtiéndolo en $\mathrm{O}_{2}$ y la enzima GPX convirtiendo el $\mathrm{H}_{2} \mathrm{O}_{2}$ en $\mathrm{H}_{2} \mathrm{O}$ (figura 2).

En el ciclo del glutatión-ascorbato, el peróxido de hidrógeno es convertido a agua, el agente reductor en la primera reacción catalizada por la APX es el ascorbato (AsA) el cual se oxida en monodehidroascorbato (MDA). Posteriormente, la MDA reductasa (MDAR) reduce el MDA a ascorbato con la ayuda del NAD(P)H. El dehidroascorbato (DHA) se produce espontáneamente por MDA y puede ser reducido a ascorbato por la DHA reductasa (DHAR) con la ayuda del glutatión (GSH) que es oxidado a glutatión oxidado (GSSG). El ciclo se cierra cuando el glutatión reductasa (GR) convierte el GSSG en $\mathrm{GSH}$ con el agente reductor $\mathrm{NAD}(\mathrm{P}) \mathrm{H}$. El ciclo del glutatión peroxidasa convierte el peróxido de hidrógeno en agua usando equivalentes de reducción del GSH, el GSSG de nuevo es convertido a GSH por GR y el agente reducido NAD(P) $\mathrm{H}$ (Apel y Hirt, 2004) (figura 2).

El aumento en la concentración de enzimas antioxidantes en la planta bajo condiciones de hipoxia es un parámetro de tolerancia al estrés por inundación. Durante hipoxia en plantas de manzana, la actividad de la SOD, la POD y la GR aumentó (Bai et al., 2010), mientras que en plantas de maíz, sometidas a inundación, se incrementó significativamente la actividad de la APX, la SOD y la CAT (Bin et al., 2010). Igualmente, especies de Dendranthema spp. tolerantes a inundación, presentaron mayores niveles de SOD, APX y CAT que las especies susceptibles (Yin et al., 2010). Se ha encontrado que las enzimas SOD y CAT muestran respuestas tempranas a la hipoxia, mientras la APX presenta una respuesta tardía (Hossain et al., 2009). El aumento de antioxidantes sugiere que el sistema de defensa actúa en conjunto para proteger a la planta del daño oxidativo causado por las ROS (Mittler, 2002). 
Bajo inundación, la capacidad de la planta para eliminar ROS disminuye y hay acumulación de $\mathrm{H}_{2} \mathrm{O}_{2}$ que causa peroxidación lipídica, incrementos en la permeabilidad de la membrana $y$ aceleración de la senescencia foliar (YongZhong et al., 2010). El malondialdehido (MDA) es el primer producto de la peroxidación lipídica de la membrana y es considerado como un indicador del daño oxidativo en la planta (Bai et al., 2010).

En genotipos de maíz tolerantes, el contenido de MDA en hojas y raíces no fue alterado por un periodo de inundación de $6 \mathrm{~d}$, en contraste con los genotipos susceptibles donde aumentó significativamente en hojas y raíces (Bin et al., 2010). En plantas de trigo sometidas a un periodo de inundación de $10 \mathrm{~d}$, el contenido de MDA en cloroplastos se incrementó (Zheng et al., 2009), mientras que en cítricos se encontró que el nivel de MDA incrementó continuamente a medida que aumentaba el periodo de inundación (Hos- sain et al., 2009). En plantas de Dendranthema spp. susceptibles a inundación, el contenido de MDA es mayor que en plantas tolerantes (Yin et al., 2010). Una disminución en la actividad de las enzimas antioxidantes puede resultar en la acumulación de altos niveles de $\mathrm{H}_{2} \mathrm{O}_{2}$ y en un incremento en la peroxidación de lípidos, de tal modo que aumenta el contenido de MDA y se induce daño en membranas (Bin et al., 2010). Los cambios en la actividad de las enzimas antioxidantes y el contenido de MDA son muy variables entre especies y dependen en gran medida de la duración del estrés (Bin et al., 2010).

Durante un periodo corto de inundación, la regulación del sistema antioxidante es una respuesta de protección contra ROS; no obstante, durante un periodo de inundación prolongada el mecanismo regulador del estrés no tiene la capacidad para reparar el daño oxidativo y como consecuencia ocurre la peroxidación lipídica (Balakhina et al., 2010).

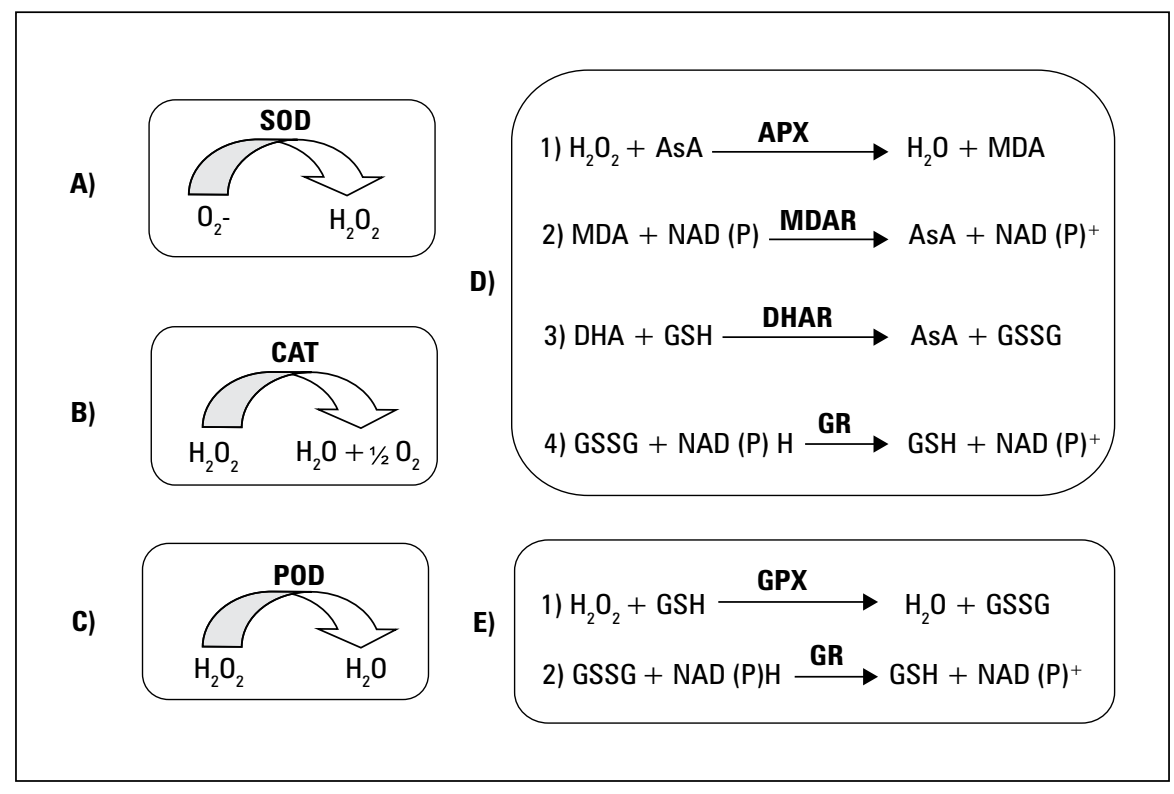

Figura 2. Principales mecanismos de detoxificación de ROS por: a) la superóxido dismutasa (SOD), b) la catalasa (CAT), c) la peroxidasa (POD), d) el ciclo del ascorbato-glutatión y e) el ciclo del glutatión peroxidasa. 


\section{ADAPTACIONES MORFOLÓGICAS Y ANATÓMICAS}

Después del ajuste metabólico, la planta genera cambios morfológicos internos para sobrevivir bajo largos periodos de hipoxia. Kolb y Joly (2009) señalan que en plantas de Tabebuia cassinoides la disminución en la producción de etanol, después de unos días de inundación, coincidió con la aparición de raíces nuevas.

En plantas de álamo (Populus deltoides Marsh.), como respuesta a la inundación hay formación de lenticelas hipertrofiadas y generación de raíces adventicias. Gradualmente, los tallos sumergidos comienzan a hincharse después de $10 \mathrm{~d}$ de inundación, las lenticelas hipertrofiadas aparecen de 10 a 15 d y las raíces adventicias a los 20 d de inundación (Cao y Conner, 1999). Igualmente en plantas de Himatanthus sucuuba se induce la formación de lenticelas hipertrofiadas y raíces adventicias $15 \mathrm{~d}$ después de iniciarse la inundación (Ferreira et al., 2009). Las lenticelas son estructuras que facilitan el transporte de oxígeno a las raíces, contribuyendo a la recuperación y mantenimiento de la respiración aeróbica en plantas sometidas a inundación (Ferreira et al., 2009), además ayudan en la liberación de compuestos tóxicos (Cao y Conner, 1999).

El principal cambio a nivel morfológico en condiciones de inundación, es la formación de aerénquima, un tejido vegetal con espacios largos e interconectados llenos de gas (Koppitz, 2004). La formación de aerénquima es específica de algunas especies y puede diferir entre genotipos dentro de una misma especie (Colmer, 2003). El aerénquima ofrece a la planta una vía interna de baja resistencia para el movimiento de oxígeno (Colmer et al., 2006; Aschi-Smiti, 2003), facilitando su transporte desde la parte aérea hasta la raíz (Aschi-Smiti, 2003; Kolb y Joly, 2009).

El oxígeno que llega a la raíz vía aerénquima mejora significativamente el estado energético de la planta, permitiendo la sobrevivencia en ausencia de oxígeno externo (Aschi-Smiti, 2003). Segmentos de raíces adventicias de plantas crecidas durante $15 \mathrm{~d}$ en condiciones de hipoxia presentan mayor AEC (carga energética del adenilato) que segmentos de plantas crecidas aeróbicamente (Aschi-Smiti, 2003).

Cuando el aerénquima es abundante en la raíz, la planta puede presentar metabolismo aeróbico (Blom, 1999). En plantas de canola durante un periodo de inundación prolongada se forman raíces laterales pequeñas, carnosas y porosas, con alto contenido de aerénquima (Zhou y Lin 1995). En trébol blanco (Trifolium repens), Huber et al. (2009) encontraron que bajo condiciones de inundación la porosidad de la raíz incrementa $60 \%$ en comparación con condiciones de buen drenaje. Igualmente, las raíces de plantas de trigo que son expuestas a inundación presentan de cuatro a cinco veces más porosidad que las de plantas crecidas en suelos bien drenados (Malik et al., 2003).

El desarrollo de aerénquima también facilita el movimiento de gases desde el tejido a la atmósfera, siendo un proceso importante para la detoxificación y eliminación de gases como metano y etileno que se acumulan en cantidades tóxicas bajo condiciones de estrés por inundación (Colmer, 2003).

El aerénquima puede ser constitutivo en especies tolerantes a inundación como el arroz (Oriza sativa L.) o su formación puede ser inducida por factores como presencia de zonas deficientes de $\mathrm{O}_{2}$, el transporte interno de ácido 1-aminociclopropano-1-carboxílico, precursor del etileno, o la acumulación de etileno en el suelo (Malik et al., 2003).

En general, las especies que tienen aerénquima constitutivo presentan ventaja competitiva en condiciones de inundación frente a las especies que no lo tienen. Las especies de tierras húmedas que presentan aerénquima constitutivo son capaces de incrementar la porosidad rápidamen- 
te cuando se exponen a condiciones deficientes de oxígeno (Visser et al., 2000). Igualmente, los genotipos que producen raíces secundarias, en condiciones de buen drenaje, tienden a ser más productivos durante la inundación que los genotipos que producen pocas o ninguna raíz en condiciones óptimas de crecimiento (Huber et al., 2009).

Además, el aerénquima ayuda al crecimiento y producción de raíces en condiciones de anoxia (Aguilara et al., 1999; Malik et al., 2003). Bajo inundación también se incrementa la proporción de raíces nodales con aerénquima, facilitando la difusión de $\mathrm{O}_{2}$ y la elongación de la raíz (Suralta y Yamauchi, 2008). Las especies con alta porosidad en la raíz forman raíces más profundas que mejoran la exploración del suelo durante la inundación y ofrecen una ventaja competitiva en el crecimiento, al finalizar el periodo de inundación (Colmer, 2003). El aerénquima se desarrolla mejor en raíces localizadas en el agua, arriba de la superficie del suelo que en raíces ubicadas dentro del suelo (Polthanee et al., 2008). Factores morfológicos y anatómicos como la formación de aerénquima en las raíces y el desarrollo de raíces adventicias son considerados como una de las principales respuestas de las plantas para sobrevivir a largos periodos de anoxia (Aschi-Smiti, 2003; Malik et al., 2003; Saqib et al., 2005; Mano et al., 2006; Suralta y Yamauchi, 2008).

El aerénquima puede ser esquizogénico, desarrollado como resultado de la separación de las células (Kolb y Joly, 2009) o lisogénico, producido por muerte celular (Evans, 2003). El esquizogénico es usualmente constitutivo, mientras que el lisogénico es generalmente inducido y presenta una estructura menos regular (Evans, 2003). La muerte selectiva de las células para formar aerénquima está asociada con el aumento de la actividad de enzimas proteolíticas (Aschi-Smiti, 2003) o con la acumulación de etileno (Colmer, 2003). Especies tolerantes a inundación del género Dendranthema presentan aerénquima lisogénico, mientras que los genotipos susceptibles carecen de este (Yin et al., 2010), indicando que este rasgo puede ser importante en la tolerancia a inundación (Mano et al., 2006).

Otro mecanismo desarrollado por algunas plantas para hacer eficiente el uso del oxígeno bajo inundación es la formación de una barrera que impide la pérdida radial de oxígeno (ROL) desde la raíz a la rizósfera (Colmer, 2003). La barrera para impedir la ROL en la zona basal de la raíz puede mejorar la difusión longitudinal de $\mathrm{O}_{2}$ desde la parte aérea hacia el ápice de la raíz (Colmer, 2003). Igualmente, la ROL inhibe la metanogénesis, promueve la oxidación del metano $\left(\mathrm{CH}_{4}\right)$ y reduce el flujo potencial de $\mathrm{CH}_{4}$ (Li et al., 2006).

La barrera que evita la ROL es producto de una resistencia física (componente dominante), causada por una pared secundaria en las capas de la hipodermis, y de la actividad respiratoria en la capa hipodermis/epidermis (Garthwaite et al., 2008). Plantas como Hordeum marinum forman lignina, suberina y otros compuestos fenólicos en la pared de las células bajo condiciones de inundación (Garthwaite et al., 2008). En plantas de Imatanthus sucuuba hay cutinización de la pared celular (Ferreira et al., 2009), lo que lleva a la formación de la barrera como un mecanismo para evitar la pérdida del $\mathrm{O}_{2}$ en la raíz. La permeabilidad del $\mathrm{O}_{2}$ a través de la capa externa de células radicales, generada por la barrera, fue reducida en promedio 27 veces en plantas sometidas a inundación, en comparación con las plantas crecidas en condiciones aeróbicas (Garthwaite et al., 2008).

En plantas de Hordeum marinum que crecieron bajo anoxia, la estela ocupa $26 \%$ del total de una sección del corte de la raíz (Garthwaite et al., 2008), en Vetiveria filipes, el porcentaje que ocupa la estela es de 27,3 en suelos aireados y 20,2 en suelos estancados, en Sorghum bicolor es de 35,5 y 26,4 respectivamente, en Cenchrus ciliaris el porcentaje de la estela es de 33,3 y 24,9 en suelos aireados y anegados respectivamente (McDonald et al., 2002), mientras que en plantas de álamo 
(Populus deltoides Marsh.) el tallo sumergido fue más delgado que el de las plántulas en condiciones de normoxia (Cao y Conner, 1999), estas modificaciones le permiten a la planta tener menores tasas de agotamiento de $\mathrm{O}_{2}$ en la raíz. En plántulas de Tabebuia cassinoides sometidas a inundación hay cambios en la morfología radicular formándose nuevas raíces de forma rectilínea y menos ramificada (Kolb y Joly, 2009), lo que le permite explorar mayor área en busca de oxígeno en el suelo.

En manglares (Avicennia marina [Forsk.] Vierh.) hay un aumento en el diámetro de tallo, el grosor del córtex y el diámetro de la médula a medida que aumenta el periodo de inundación, mientras que el diámetro tangencial de los vasos, el grosor de la pared y el grosor de los vasos disminuyen significativamente (Xiao et al., 2009). Esto puede relacionarse con un mecanismo de reducción de los espacios intercelulares para evitar la pérdida de oxígeno, así mismo Colmer (2003) señala que las raíces con el diámetro de la estela más grande tienden a tener mayores tasas de agotamiento de $\mathrm{O}_{2}$.

Usualmente, los árboles sometidos a estrés por inundación pueden abastecer la demanda de $\mathrm{O}_{2}$ para la respiración de sus raíces vía lenticelas en los tallos, sistemas conductores de aire o vía neumatóforos (Parolin, 2009). Los neumatóforos son raíces que crecen verticalmente desde el suelo inundado hacia la superficie en busca de aire, son epigeas con geotropismo negativo, provistas de aberturas parecidas a lenticelas cuya función es proporcionar aire a las raíces que se encuentran en lugares pantanosos (Curran, 1985; García, 2007).

Cuando los neumatóforos son completamente cubiertos por agua, la presión interna de gas disminuye como consecuencia del consumo de oxí- geno en la respiración de las células radiculares (Medina, 1999). La presión desarrollada en este proceso es alrededor de 0,5 bares, pero el agua no puede entrar a los neumatóforos porque la presión hidrostática no es suficiente para romper la tensión superficial a este nivel. A bajos niveles del agua, el aire penetra fácilmente a través de los poros suministrando oxígeno para la respiración celular (Medina, 1999).

Además de los neumatóforos, las árboles en los bosques húmedos tropicales pueden generar otras adaptaciones morfológicas que favorecen la difusión de oxígeno aéreo a la raíz como las raíces tablares y fúlcreas (Parolin y Wittman, 2010). Las tablares son compactas, amplias y expandidas en formas de láminas. Las fúlcreas son como "anclas" que crecen desde el tallo arqueándose hacia el suelo. Tanto los neumatóforos como las raíces tablares se forman por las condiciones de alta humedad del suelo y por la poca acumulación de materia orgánica (García, 2007).

\section{CONCLUSIONES Y PERSPECTIVAS}

La investigación de los mecanismos de tolerancia a estrés hídrico por inundación en plantas se ha convertido en un objetivo importante en la agricultura, debido a las recientes olas de inundación presentadas en diferentes zonas del mundo. Entender cómo la planta responde a la deficiencia de oxígeno es básico para los programas de mejoramiento que buscan crear plantas capaces de tolerar ciertos periodos de anegamiento, esto se plantea como una necesidad imperativa para hacer frente al cambio climático. Es importante conocer los rasgos fisiológicos que expresan las plantas sometidas a inundación y los genes que regulan la expresión de dichos rasgos, con el fin de entender cuál es la base de las diferencias entre las plantas tolerantes y sensibles y entre especies. 


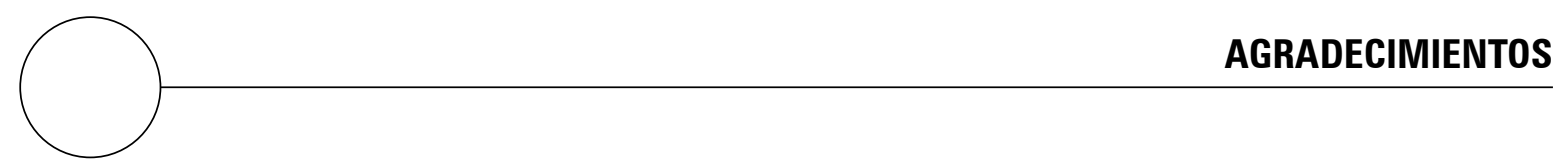

Agradecemos a Juan Andrés Cardoso Arango por sus comentarios sobre el artículo.

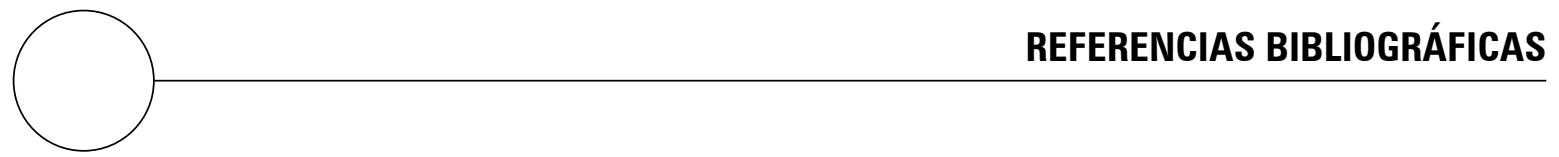

Abbaspour, A. 2012. Fractionation of copper in soils as influenced by waterlogging and application of crop residues. Iranian J. Soil Res. (Soil and Water Sci.) 25(4), 295-306.

Aguilara, E.A., D.W. Turnera y K. Sivasithamparam. 1999. Aerenchyma formation in roots of four banana (Musa spp.) cultivars. Sci. Hortic. 80, 57-72.

Ahmed, S., E. Nawat y T. Sakuratani. 2006. Changes of endogenous $\mathrm{ABA}$ and $\mathrm{ACC}$, and their correlations to photosynthesis and water relations in mungbean (Vigna radiata (L.) Wilczak cv. KPS1) during waterlogging. Environ. Exp. Bot. 57, 278-284.

Alscher, R.G., J.L. Donahue y C.L. Cramer. 1997. Reactive oxygen species and antioxidants: Relationships in green cells. Physiol. Plant. 100, 224-233.

Apel, K. y H. Hirt. 2004. Reactive oxygen species: Metabolism, oxidative stress, and signal transduction. Annu. Rev. Plant Biol. 55, 373-99.

Aschi-Smiti, S., W. Chaièbi, R. Brouquisse, B. Ricard y P. Saglio. 2003. Assessment of enzyme induction and aerenchyma formation as mechanisms for flooding tolerance in Trifolium subterraneum 'Park'. Ann. Bot. 91, 195-204.

Bai, T., C. Li, F. Ma, F. Feng y H. Shu. 2010. Responses of growth and antioxidant system to root-zone hypoxia stress in two Malus species. Plant Soil 327, 95-105.

Balakhnina, T.I., P. Bennicelli, Z. Stepniewska, W. Stepniewski e I.R. Fomina. 2010. Oxidative damage and antioxidant defense system in leaves of Vicia faba major L. cv. Bartom during soil flooding and subsequent drainage. Plant Soil 327, 293-301.

Bange, M.P., S.P. Milroy y P. Thongbai. 2003. Growth and yield of cotton in response to waterlogging. Field Crops Res. 88, 129-142.

Bin, T., X. Shang-Zhong, Z. Xi-Ling, Z. Yong-Lian y O. Fa-Zhan. 2010. Changes of antioxidative enzymes and lipid peroxidation in leaves and roots of waterlogging-tolerant and waterlogging-sensitive maize genotypes at seedling stage. Agr. Sci. China 9(5), 651-661.

Blom, C.W.P.M. 1999. Adaptations to flooding stress: From plant community to molecule. Plant Biol. 1, 261-273.

Cao, F.L. y W.H. Conner. 1999. Selection of flood-tolerant Populus deltoides clones for reforestation projects in China. For. Ecol. Manage. 117, 211-220

Cheeseman, J.M. 2007. Hydrogen peroxide and plant stress: A challenger relationship. Plant Stress 1(1), 4-15.

Chen, H., R. G. Qualls y G.C. Miller. 2002. Adaptive responses of Lepidium latifolium to soil flooding: biomass allocation, adventitious rooting, aerenchyma formation and ethylene production. Environ. Exp. Bot. 48, 119-128.

Colmer, T.D. 2003. Long-distance transport of gases in plants: a perspective on internal aeration and radial oxygen loss from roots. Plant Cell Environ. 26 17-36.

Colmer, T.D., M.C.H. Cox y L.A.C.J. Voesenek. 2006. Root aeration in rice (Oryza sativa): evaluation of oxygen, carbon dioxide, and ethylene as possible regulators of root acclimatizations. New Phytol. $170,767-778$.

Couldwell, D.L., R. Dunford, N.J. Kruger, D.C. Lloyd, R.G. Ratcliffe y A.M.O. Smitdh. 2009. Response of cytoplasmic $\mathrm{pH}$ to anoxia in plant tissues with altered activities of fermentation enzymes: application of methyl phosphonate as an NMR pH probe. Ann. Bot. 103, 249-258.

Curran, M. 1985. Gas movements in the roots of Avicennia marina (Forsk.) Vierh. Aust. J. Plant Physiol. 12, 97-108.

Dickin, E. y D. Wright. 2007. The effects of winter waterlogging and summer drought on the growth and 
yield of winter wheat (Triticum aestivum L.). Eur. J. Agron. 28, 234-244.

Ella, E.S., N. Kawano y O. Ito. 2003. Importance of active oxygen-scavenging system in the recovery of rice seedlings after submergence. Plant Sci. 165, 85-93.

Evans, D.E. 2003. Aerenchyma formation. New Phytol. $161,35-49$.

Ferreira, C.S., M.T.F. Piedade, A.C. Franco, J.F. Carvalho Goncalves y W.J. Junk. 2009. Adaptive strategies to tolerate prolonged flooding in seedlings of flood plain and upland populations of Himatanthus sucuuba, a Central Amazon tree. Aquatic Bot. 90, 246-252.

Fukao, T., R.A. Kennedy, Y. Yamasue y M.E. Rumpho. 2003. Genetic and biochemical analysis of anaerobically induced enzymes during seed germination of Echinochloa crus-galli varieties tolerant and intolerant of anoxia. J. Exp. Bot. 54, 1421-1429.

Fukao, T. y J. Bailey-Serres. 2004. Plant responses to hypoxia - is survival a balancing act?. Trends Plant Sci. 9(9), 490-494.

García, FJ. 2007. Biología y botánica, Tema 6b: Modificaciones y adaptaciones de la raíz. Unidad docente de botánica. ETSMRE, Universidad Politécnica de Valencia, Valencia, España.

García, P.N. y F. Aldana. 2011. Efecto del estrés por anegamiento sobre el crecimiento, desarrollo y fisiología de uchuva (Physalis peruviana L.) bajo condiciones de invernadero. Trabajo de grado. Facultad de Agronomía, Universidad Nacional de Colombia, Bogotá.

Garthwaite, A.J., W. Armstrong y T.D. Colmer. 2008. Assessment of $\mathrm{O}_{2}$ diffusivity across the barrier to radial $\mathrm{O}_{2}$ loss in adventitious roots of Hordeum marinum. New Phytol. 179, 405-416.

Grassini, P., G.V. Indaco, M. López, A.J. Hall y N. Trápani. 2006. Responses to short-term waterlogging during grain filling in sunflower. Field Crops Res. 101, 352-363.

Guglielminetti, L., H.A. Busilacchi, P. Perata y A. Alpi. 2001. Carbohydrate-ethanol transition in cereal grains under anoxia. New Phytol. 151, 607-612.

Gutierrez, B.F.H., R.S. Lavado y C.A. Porcelli. 1996. Note on the effects of winter and spring waterlogging on growth, chemical composition and yield of rapeseed. Field Crops Res. 47, 175-179.

Hossain, Z., M.F. López-Climent, V. Arbona, R.M. PérezClemente y A. Gómez-Cadenas. 2009. Modulation of the antioxidant system in citrus under waterlog- ging and subsequent drainage. J. Plant Physiol. 166, 1391-1404.

Huber, H., E. Jacobs y E.J.W. Visser. 2009. Variation in flooding-induced morphological traits in natural populations of white clover (Trifolium repens) and their effects on plant performance during soil flooding. Ann. Bot. 103, 377-386.

IPCC. 2007. Climate change 2007: Contribution of working group I to the fourth assessment report of the intergovernmental panel on climate change. Cambridge University Press, Cambridge, UK.

Issarakraisila, M., Q. Ma y D.W. Turner. 2006. Photosynthetic and growth responses of juvenile Chinese kale (Brassica oleracea var. alboglabra) and Caisin (Brassica rapa subsp. parachinensis) to waterlogging and water deficit. Sci. Hortic. 111, 107-113

Kato-Noguchi, H. 2000. Evaluation of the importance of lactate for the activation of ethanolic fermentation in lettuce roots in anoxia. Physiol. Plant. 109, 28-33.

Kolb, R.M. y C.A. Joly. 2009. Flooding tolerance of Tabebuia cassinoides: Metabolic, morphological and growth responses. Flora 204, 528-535.

Koppitz, H. 2004. Effects of flooding on the amino acid and carbohydrate patterns of Phragmites australis. Limnologica 34, 37-47.

Kulichikhin, K.Y., T.V. Chirkova y K. Fagerstedt. 2008. Intracellular $\mathrm{pH}$ in rice and wheat root tips under hypoxic and anoxic conditions. Plant Signal. Behav. 3, 240-242.

Leul, M. y W. Zhou. 1998. Alleviation of waterlogging damage in winter rape by application of uniconazole: Effects on morphological characteristics, hormones and photosynthesis. Field Crops Res. 59, 121-127.

Li, S., S.R. Pezeshki y F.D. Shields. 2006. Partial flooding enhances aeration in adventitious roots of black willow (Salix nigra) cuttings. J. Plant Physiol. 163, 619-628.

Lin, K.H., P.Y. Chao, S.Y. Yang, W.C. Chen, H.F Lo y T.R Chang. 2006. The effects of flooding and drought stresses on the antioxidant constituents in sweet potato leaves. Bot. Studies 47, 417-426.

Lin, K.H., Y.K. Chiou, S.Y. Hwang, L.F.O. Chen y H.F. Lo. 2008. Calcium chloride enhances the antioxidative system of sweet potato (Ipomoea batatas) under flooding stress. Ann. Appl. Biol. 152, 157-168.

Malik, A.I., T.D. Colmer, H. Lambers y M. Schortemeyer. 2003. Aerenchyma formation and radial $\mathrm{O}_{2}$ loss along adventitious roots of wheat with only the 
apical root portion exposed to $\mathrm{O}_{2}$ deficiency. Plant Cell Environ. 26, 1713-1722.

Mano, Y., F. Omori, T. Takamizo, B. Kindiger, R. McBird y C.H. Loaisiga. 2006. Variation for root aerenchyma formation in flooded and non-flooded maize and teosinte seedlings. Plant Soil 281, 269-279.

Medina, E. 1999. Mangrove physiology: the challenge of salt, heat, and light stress under recurrent flooding, pp. 109-126. En: Yáñez-Arancibia, A. y A.L. LaraDomínguez (eds.). Ecosistemas de Manglar en América Tropical. Instituto de Ecología, México; UICN/ORMA, Costa Rica; NOAA/NMFS, Silver Spring, MD.

McDonald, M.P., N.W. Galwey y T.D. Colmer. 2002. Similarity and diversity in adventitious root anatomy as related to root aeration among a range of wetland and dryland grass species. Plant Cell Environ. $25,441-451$.

Milroy, S.P., M.P. Bange y P. Thongbai. 2009. Cotton leaf nutrient concentrations in response to waterlogging under field conditions. Field Crops Res. 113, 246-255

Mittler, R. 2002. Oxidative stress, antioxidants and stress tolerance. Trends Plant Sci. 7(9), 405-410.

Mohanty, B. y B. Ong. 2001. Contrasting effects of submergence in light and dark on pyruvate decarboxylase activity in roots of rice lines differing in submergence tolerance. Ann. Bot. 91, 291-300.

Mubarakshina, M., S. Khorobrykh y B. Ivanov. 2006. Oxygen reduction in chloroplast thylakoids results in production of hydrogen peroxide inside the membrane. Biochim. Biophys. Acta 1757, 1496-1503.

Palta, J.A., A. Ganjeali, N.C. Turner, K.H.M. Siddique. 2010. Effects of transient subsurface waterlogging on root growth, plant biomass and yield of chickpea. Agr. Water Manage. 97, 1469-1476.

Parolin, P. 2009. Submerged in darkness: adaptations to prolonged submergence by woody species of the Amazonian floodplains. Ann. Bot. 103, 359-376.

Parolin, P. y F. Wittman. 2010. Struggle in the flood: tree responses to flooding stress in four tropical floodplain systems. En: http://www.ncbi.nlm.nih.gov/ pmc/articles/PMC29650 40/pdf/plq003.pdf; consulta: mayo de 2011.

Peña-Fronteras, J.T., M.C. Villalobos, A.M. Baltazar, F.E. Merca, A.M. Ismail y D.E. Johnson. 2008. Adaptation to flooding in upland and lowland ecotypes of Cyperus rotundus, a troublesome sedge weed of rice: tuber morphology and carbohydrate metabolism. Ann. Bot. 103, 295-302.

Polthanee, A., T. Changdee, J. Abe y S. Morita. 2008 Effects of flooding on growth, yield and aerenchyma development in adventitious roots in four cultivars of Kenaf (Hibiscus cannabinus L.). Asian J. Plant Sci. 7(6), 544-550.

Ryser, P., K.G. Harneet y J.B. Collin. 2011. Constraints of root response to waterlogging in Alisma triviale. Plant Soil 343, 247-260.

Sairam, R.K., K. Dharmar, V. Chinnusamy y R.C. Meena. 2008. Waterlogging-induced increase in sugar mobilization, fermentation, and related gene expression in the roots of mung bean (Vigna radiata). J. Plant Physiol. 166, 602-616.

Saqib, M., J. Akhtar y R.H. Qureshi. 2005. $\mathrm{Na}^{+}$exclusion and salt resistance of wheat (Triticum aestivum) in saline-waterlogged conditions are improved by the development of adventitious nodal roots and cortical root aerenchyma. Plant Sci. 169, 125-130.

Stryer, L. 1995. Bioquímica. $4^{a}$ ed. Editorial Reverté S.A., Barcelona, España.

Suralta, R. y A. Yamauchi A. 2008. Root growth, aerenchyma development, and oxygen transport in rice genotypes subjected to drought and waterlogging. Environ. Exp. Bot. 64, 75-82

Suzuki, N. y R. Mittler. 2006. Reactive oxygen species and temperature stresses: A delicate balance between signaling and destruction. Physiol. Plant. 126, 45-51.

Taiz, L. y E. Zeiger. 2010. Plant physiology. $5^{\text {th }}$ ed. Sinauer Associates, Sunderland, MA.

Unger, I.M., P.P. Motavalli y R.-M. Muzika. 2009a. Changes in soil chemical properties with flooding: A field laboratory approach. Agr. Ecosyst. Environ. $131,105-110$

Unger, I.M., A.C. Kennedy y R.M. Muzika. 2009b. Flooding effects on soil microbial communities. Appl. Soil Ecol. 42, 1-8.

Visser, E.J.W., T.D. Colmer, C.W.P.M. Blom y L.A.C.J.Voesenek. 2000. Changes in growth, porosity, and radial oxygen loss from adventitious roots of selected mono- and dicotyledonous wetland species with contrasting types of aerenchyma. Plant Cell Environ. 23, 1237-1245.

Wood, S., K. Sebastian y S. Scherr. 2000. Soil resource condition. pp. 45-54. En: Pilot analysis of global ecosystems: Agroecosystems. International Food 
Policy Research Institute and the World Resources Institute, Washington, D.C.

Xiao-Chang, W. y L. Oin. 2006. Effect of waterlogged and aerobic incubation on enzyme activities in paddy soil. Pedosphere 16(4), 532-539.

Xiao, Y., Z. Jie, M. Wanga, G. Lin y W. Wang. 2009. Leaf and stem anatomical responses to periodical waterlogging in simulated tidal floods in mangrove Avicennia marina seedlings. Aquatic Bot. 91, 231-237.

Yin, D., S. Chen, F. Chen, Z. Guan y W. Fang. 2009. Morphological and physiological responses of two chrysanthemum cultivars differing in their tolerance to waterlogging. Environ. Exp. Bot. 67, 87-93.

Yin, D., S. Chen, F. Chen, Z. Guan y W. Fang. 2010. Morpho-anatomical and physiological responses of two Dendranthema species to waterlogging. Environ. Exp. Bot. 68, 122-130.

Yordanova, R., K.N. Christov, L.P. Popova. 2004. Antioxidative enzymes in barley plants subjected to soil flooding. Environ. Exp. Bot. 51, 93-101.
Yong, C., G. Min, C. Ye, Z. Chong-Shun, Z. Xue-Kun y W. Han-Zhong. 2010. Combining ability and genetic effects of germination traits of Brassica napus 1. under waterlogging stress condition. Agr. Sci. China 9(7), 951-957.

Yong-Zhong L., T. Bin, Z. Yong-Lian, M. Ke-Jun, X. ShangZhong y Q. Fa-Zhan. 2010. Screening methods for waterlogging tolerance at maize (Zea mays L.) seedling stage. Agr. Sci. China 9(3), 362-369.

Zaidi, P.H., S. Rafique y N.N. Singh. 2002. Response of maize (Zea mays L.) genotypes to excess soil moisture stress: morpho-physiological effects and basis of tolerance. Eur. J. Agron. 19, 383-399.

Zheng, C., D. Jiang, F. Liu, T. Dai, O. Jing y W. Cao. 2009. Effects of salt and waterlogging stresses and their combination on leaf photosynthesis, chloroplast ATP synthesis, and antioxidant capacity in wheat. Plant Sci. 176, 575-582.

Zhou, E. y X. Lin. 1995. Effects of waterlogging at different growth stages on physiological characteristics and seed yield of winter rape (Brassica napus L.). Field Crops Res. 44, 103-110. 